ANNALES

POLONICI MATHEMATICI

$91.2-3(2007)$

\title{
Reproducing kernels for holomorphic functions on some balls related to the Lie ball
}

by Keiko Fujita (Saga)

\begin{abstract}
We consider holomorphic functions and complex harmonic functions on some balls, including the complex Euclidean ball, the Lie ball and the dual Lie ball. After reviewing some results on Bergman kernels and harmonic Bergman kernels for these balls, we consider harmonic continuation of complex harmonic functions on these balls by using harmonic Bergman kernels. We also study Szegö kernels and harmonic Szegő kernels for these balls.
\end{abstract}

Introduction. In the complex Euclidean space $\mathbb{C}^{n+1}$ we consider the function

$$
N_{p}(z)=\left(\frac{\left(\|z\|^{2}+\sqrt{\|z\|^{4}-\left|z^{2}\right|^{2}}\right)^{p / 2}+\left(\|z\|^{2}-\sqrt{\|z\|^{4}-\left|z^{2}\right|^{2}}\right)^{p / 2}}{2}\right)^{1 / p},
$$

where $\|z\|^{2}=z_{1} \bar{z}_{1}+\cdots+z_{n+1} \bar{z}_{n+1}$ and $z^{2}=z_{1}^{2}+\cdots+z_{n+1}^{2}$ for $z=$ $\left(z_{1}, \ldots, z_{n+1}\right) \in \mathbb{C}^{n+1}$. In [10], we showed that $N_{p}(z)$ is a norm on $\mathbb{C}^{n+1}$ if $p \geq 1$. Note that $N_{2}(z)=\|z\|$ is the complex Euclidean norm. By using these norms we define $N_{p}$-balls (see Section 1). Locally any holomorphic function can be expanded into a double series by means of homogeneous harmonic polynomials. In [6], we characterized holomorphic functions on the $N_{p}$-balls by the growth behavior of homogeneous harmonic polynomials. Then we studied Bergman kernels for the $N_{p}$-balls in [2], [4] and [5]. In general, explicit forms of Bergman kernels are known only for some special domains. For the $N_{p}$-balls, explicit forms are known only for the $N_{1}$-ball (the dual Lie ball), the $N_{2}$-ball (the Euclidean ball) and the $N_{\infty}$-ball (the Lie ball). However in [2] we were able to express the Bergman kernel for any $N_{p^{-}}$-ball by a double series expansion (see Section 3 ).

J. Siciak [13] showed that harmonic functions on the real ball can be continued analytically to the Lie ball. On the other hand, they are in one-to-one

2000 Mathematics Subject Classification: 32A25, 30E20, 31B10.

Key words and phrases: integral representation, reproducing kernel, Bergman kernel, Szegö kernel. 
correspondence with hyperfunctions on the sphere under the boundary value operator. Therefore we are interested in complex harmonic functions, and we have considered the harmonic Bergman kernel which is the reproducing kernel of the Hilbert space of complex harmonic functions on the $N_{p}$-balls in [3] and [5] (see Section 3).

In this paper, we will prove that harmonic functions on the $N_{p}$-ball with radius $r$ can be continued harmonically to the Lie ball with radius $2^{1 / p} r$ by using the harmonic Bergman kernel (Theorem 3.4).

So far we have not considered the Szegö kernels and harmonic Szegö kernels for the $N_{p}$-balls but it is easy to see that results similar to those on the Bergman kernels and harmonic Bergman kernels hold for the Szegö kernels and harmonic Szegö kernels, respectively. In Section 4, we will represent the Szegö kernels and the harmonic Szegö kernels by double series expansions (Theorems 4.1 and 4.2) although explicit forms of the Szegö kernels are known only for the Euclidean ball and the dual Lie ball.

In Section 5, we treat the two-dimensional case. The $N_{p}$-norm is then equivalent to the $L_{p}$-norm, and J.-D. Park [12] obtained the Bergman kernels and the Szegö kernels in explicit form for $p=4,4 / 3$. By employing his results and an idea of Bell [1], we are able to get the harmonic Bergman kernels and the harmonic Szegö kernels in explicit form for $p=4,4 / 3$ in addition to $p=1,2, \infty$.

The author would like to thank Professor Mitsuo Morimoto for useful discussion.

1. $N_{p}$-ball. First we review the $N_{p}$-norms defined by (1) (see [10] for the details). For later convenience, we put

$$
\begin{aligned}
L(z, w) & =z \cdot w+\sqrt{(z \cdot w)^{2}-z^{2} w^{2}}, \\
M(z, w) & =z \cdot w-\sqrt{(z \cdot w)^{2}-z^{2} w^{2}},
\end{aligned}
$$

where $z \cdot w=z_{1} w_{1}+\cdots+z_{n+1} w_{n+1}$. Then the $N_{p}$-norm is represented by

$$
N_{p}(z)=\left(\frac{L(z, \bar{z})^{p / 2}+M(z, \bar{z})^{p / 2}}{2}\right)^{1 / p} .
$$

For $p \geq 1, N_{p}(z)$ is an increasing function of $p$ :

$$
N_{1}(z) \leq N_{s}(z) \leq N_{2}(z) \leq N_{t}(z), \quad 1<s<2<t,
$$

and $\lim _{p \rightarrow \infty} N_{p}(z)=L(z)$, where

$$
\begin{aligned}
L(z) & =\sqrt{\|z\|^{2}+\sqrt{\|z\|^{4}-\left|z^{2}\right|^{2}}} \\
& =\inf \left\{\sum_{j=1}^{m}\left|a_{j}\right|\left\|x_{j}\right\| ; z=\sum_{j=1}^{m} a_{j} x_{j}, x_{j} \in \mathbb{R}^{n+1}, a_{j} \in \mathbb{C}, m \in \mathbb{Z}_{+}\right\}
\end{aligned}
$$


is the Lie norm. By using the notation (2), we have

$$
L(z)^{2}=\max \{L(z, \bar{z}), M(z, \bar{z})\} .
$$

Note that $N_{1}(z)=\sqrt{\left(\|z\|^{2}+\left|z^{2}\right|\right) / 2}=L^{*}(z)=\sup \{|z \cdot w| ; L(w) \leq 1\}$ is the dual Lie norm.

Define the open $N_{p^{-}}$ball $\widetilde{B}_{p}(r)$ by

$$
\widetilde{B}_{p}(r)=\left\{z \in \mathbb{C}^{n+1} ; N_{p}(z)<r\right\},
$$

and set

$$
\widetilde{B}(r)=\widetilde{B}_{\infty}(r)=\bigcap_{p \geq 1} \widetilde{B}_{p}(r)=\left\{z \in \mathbb{C}^{n+1} ; L(z)<r\right\} .
$$

By the definition, $\widetilde{B}_{p}(r)$ is an open convex and balanced subset of $\mathbb{C}^{n+1}$. In particular, $\widetilde{B}_{p}(r)$ is a domain of holomorphy in $\mathbb{C}^{n+1}$. Since $2^{-1 / p} L(z) \leq$ $N_{p}(z) \leq L(z)$, we have $\widetilde{B}(r) \subset \widetilde{B}_{p}(r) \subset \widetilde{B}\left(2^{1 / p} r\right)$.

2. Complex harmonic functions. We denote by $\mathcal{O}\left(\widetilde{B}_{p}(r)\right)$ the space of holomorphic functions on $\widetilde{B}_{p}(r)$ equipped with the topology of uniform convergence on compact sets, and by $\mathcal{O}\left(\widetilde{B}_{p}[r]\right)$ the space of germs of holomorphic functions on the closed $N_{p}$-ball

$$
\widetilde{B}_{p}[r]=\left\{z \in \mathbb{C}^{n+1} ; N_{p}(z) \leq r\right\}
$$

equipped with the inductive limit locally convex topology. If $f$ satisfies the differential equation

$$
\Delta_{z} f(z) \equiv\left(\frac{\partial^{2}}{\partial z_{1}^{2}}+\cdots+\frac{\partial^{2}}{\partial z_{n+1}^{2}}\right) f(z)=0
$$

then $f$ is called a complex harmonic function or a harmonic function. Put

$$
\begin{gathered}
\mathcal{O}_{\Delta}\left(\widetilde{B}_{p}(r)\right)=\left\{f \in \mathcal{O}\left(\widetilde{B}_{p}(r)\right) ; \Delta_{z} f(z)=0\right\}, \\
\mathcal{O}_{\Delta}\left(\widetilde{B}_{p}[r]\right)=\left\{f \in \mathcal{O}\left(\widetilde{B}_{p}[r]\right) ; \Delta_{z} f(z)=0\right\} .
\end{gathered}
$$

For harmonic functions on the Lie ball, the Poisson integral representation is well-known. We denote by

$$
K_{r}(z, w)=\frac{r^{2(n-1)}\left(r^{4}-z^{2} \bar{w}^{2}\right)}{\left(r^{4}-2 r^{2} z \cdot \bar{w}+z^{2} \bar{w}^{2}\right)^{(n+1) / 2}}
$$

the Poisson kernel. Then we have the following Poisson integral formula:

Formula 2.1 (Poisson formula). Let $f \in \mathcal{O}_{\Delta}(\widetilde{B}(r))$. Take $\varrho<1$ sufficiently close to 1 . Then

$$
f(w)=\int_{S_{r}} f(\varrho x) \overline{K_{r}(x / \varrho, w)} d S_{r}(x), \quad w \in \widetilde{B}(\varrho r),
$$


where the right-hand side does not depend on $\varrho$, and $d S_{r}$ is the normalized Lebesgue measure on the real sphere $S_{r}=\left\{x \in \mathbb{R}^{n+1} ;\|x\|=r\right\}$.

The Poisson formula gives the inverse mapping of the isomorphism in the following theorem:

TheOREM 2.2 (J. Siciak, [13]). The restriction mapping gives a linear topological isomorphism

$$
\mathcal{O}_{\Delta}(\widetilde{B}(r)) \stackrel{\sim}{\longrightarrow} \mathcal{A}_{\Delta}(B(r)),
$$

where $\mathcal{A}_{\Delta}(B(r))$ is the space of harmonic functions on the real ball $B(r)=$ $\left\{x \in \mathbb{R}^{n+1} ;\|x\|<r\right\}$.

This means that all harmonic functions on the real ball $B(r)$ can be continued harmonically to the Lie ball $\widetilde{B}(r)$.

The Poisson kernel $K_{r}(z, w)$ can be represented by means of homogeneous harmonic extended Legendre polynomials.

2.1. Homogeneous harmonic extended Legendre polynomials. Let $P_{k, n}(t)$ be the Legendre polynomial of degree $k$ and of dimension $n+1$. Note that $P_{k, 1}(t)$ is the Chebyshev polynomial and

$$
\begin{aligned}
& P_{k, n}(t)=\frac{k ! \Gamma(n-1)}{\Gamma(k+n-1)} \sum_{l=0}^{[k / 2]}(-1)^{l} \frac{\Gamma(k+(n-1) / 2-l)}{l !(k-2 l) ! \Gamma((n-1) / 2)}(2 t)^{k-2 l}, \quad n \geq 2, \\
& P_{k, 1}(t)=T_{k}(t)=\frac{\left(t+i \sqrt{1-t^{2}}\right)^{k}+\left(t-i \sqrt{1-t^{2}}\right)^{k}}{2} .
\end{aligned}
$$

We define the homogeneous harmonic extended Legendre polynomial of degree $k$ and of dimension $n+1$ by

$$
\widetilde{P}_{k, n}(z, w)=\left(\sqrt{z^{2}}\right)^{k}\left(\sqrt{w^{2}}\right)^{k} P_{k, n}\left(\frac{z}{\sqrt{z^{2}}} \cdot \frac{w}{\sqrt{w^{2}}}\right) .
$$

Then $\widetilde{P}_{k, n}(z, w)=\widetilde{P}_{k, n}(w, z)$ and $\Delta_{z} \widetilde{P}_{k, n}(z, w)=0$. We write $\widetilde{T}_{k}(z, w)$ for $\widetilde{P}_{k, 1}(z, w)$. Note that $\widetilde{T}_{k}(z, w)=\left(L(z, w)^{k}+M(z, w)^{k}\right) / 2$. The dimension $N(k, n)$ of the space of homogeneous harmonic polynomials of degree $k$ in $\mathbb{C}^{n+1}$ is known to be

$$
\begin{aligned}
& N(0, n)=1, \\
& N(k, 1)=2, \quad k=1,2, \ldots, \\
& N(k, n)=(2 k+n-1)(k+n-2) ! /(k !(n-1) !), \quad n \geq 2 .
\end{aligned}
$$

For $z, w \in \mathbb{C}^{n+1}$ with $L(z) L(w)<r^{2}$, the Poisson kernel can be represented by an infinite sum of $\widetilde{P}_{k, n}(z, w)$ as follows:

$$
K_{r}(z, w)=\sum_{k=0}^{\infty} \frac{N(k, n)}{r^{2 k}} \widetilde{P}_{k, n}(z, \bar{w}) .
$$




\section{Bergman kernels and harmonic Bergman kernels}

3.1. Bergman kernels in explicit form. Put

$$
H \mathcal{O}\left(\widetilde{B}_{p}(r)\right)=\left\{f \in \mathcal{O}\left(\widetilde{B}_{p}(r)\right) ; \int_{\widetilde{B}_{p}(r)}|f(w)|^{2} d V_{\widetilde{B}_{p}(r)}(w)<\infty\right\},
$$

where $d V_{\widetilde{B}_{p}(r)}$ is the normalized Lebesgue measure on $\widetilde{B}_{p}(r)$, and denote by $B_{p, r}(z, w)$ the Bergman kernel of $H \mathcal{O}\left(\widetilde{B}_{p}(r)\right)$; that is, for $f \in H \mathcal{O}\left(\widetilde{B}_{p}(r)\right)$, we have

$$
f(w)=\int_{\widetilde{B}_{p}(r)} f(z) \overline{B_{p, r}(z, w)} d V_{\widetilde{B}_{p}(r)}(z), \quad w \in \widetilde{B}(r) .
$$

In the following, we write $B_{r}(z, w)$ for $B_{\infty, r}(z, w)$. For the Euclidean ball $\widetilde{B}_{2}(r)$, the Bergman kernel is well-known:

$$
B_{2, r}(z, w)=\frac{r^{2 n+4}}{\left(r^{2}-z \cdot \bar{w}\right)^{n+2}}=\frac{r^{2 n+4}}{\left(r^{2}-(L(z, \bar{w})+M(z, \bar{w})) / 2\right)^{n+2}} .
$$

For the Lie ball $\widetilde{B}(r)$, L. K. Hua [7] obtained the explicit form

$$
\begin{aligned}
B_{r}(z, w) & =\frac{r^{4 n+4}}{\left(r^{4}-2 r^{2} z \cdot \bar{w}+z^{2} \bar{w}^{2}\right)^{n+1}} \\
& =\frac{r^{4 n+4}}{\left(r^{2}-L(z, \bar{w})\right)^{n+1}\left(r^{2}-M(z, \bar{w})\right)^{n+1}} .
\end{aligned}
$$

For the dual Lie ball $\widetilde{B}_{1}(r)$, K. Oeljeklaus, P. Pflug and E. H. Youssfi [11] obtained the explicit form (note that they call the dual Lie ball the minimal ball)

$$
B_{1, r}(z, w)=\frac{\sum_{k=0}^{[(n+1) / 2]} \frac{n ! X^{n-2 k} Y^{k}}{(2 k+1) !(n+1-2 k) !}\left(2(n+1) X-(n+1-2 k)\left(X^{2}-Y\right)\right)}{\left(X^{2}-Y\right)^{n+2}},
$$

where $X=1-z \cdot \bar{w} /\left(2 r^{2}\right)$ and $Y=z^{2} \overline{w^{2}} /\left(4 r^{4}\right)$. Note that $X^{2}-Y$

$=\left(1-\left(\frac{L(z, \bar{w})^{1 / 2}+M(z, \bar{w})^{1 / 2}}{2 r}\right)^{2}\right)\left(1-\left(\frac{L(z, \bar{w})^{1 / 2}-M(z, \bar{w})^{1 / 2}}{2 r}\right)^{2}\right)$, $N_{1}(z)=\left(L(z, \bar{z})^{1 / 2}+M(z, \bar{z})^{1 / 2}\right) / 2, N_{2}(z)^{2}=(L(z, \bar{z})+M(z, \bar{z})) / 2$ and $L(z)^{2}=\max \{L(z, \bar{z}), M(z, \bar{z})\}$.

3.2. Double series expansions of Bergman kernels. For the Euclidean ball and the Lie ball, the Bergman kernels are expanded into double series 
as follows:

$$
\begin{aligned}
B_{2, r}(z, w)= & \sum_{k=0}^{\infty} \sum_{l=0}^{[k / 2]} \frac{\Gamma((n+1) / 2)(k+n+1) ! N(k-2 l, n)}{2^{k} r^{2 k} \Gamma(k-l+(n+1) / 2) l !(n+1) !} \\
& \times\left(z^{2}\right)^{l}\left(\bar{w}^{2}\right)^{l} \widetilde{P}_{k-2 l, n}(z, \bar{w}), \\
B_{r}(z, w)= & \sum_{k=0}^{\infty} \sum_{l=0}^{[k / 2]} \frac{2 \Gamma(l+(n+3) / 2) \Gamma(k+n-l+1) N(k-2 l, n)}{r^{2 k}(n+1) ! l ! \Gamma(k+(n+1) / 2-l)} \\
& \times\left(z^{2}\right)^{l}\left(\bar{w}^{2}\right)^{l} \widetilde{P}_{k-2 l, n}(z, \bar{w}) .
\end{aligned}
$$

Put

$$
\beta_{k, l, r}^{n, p}=\int_{\widetilde{B}_{p}(r)}\left|\left(\zeta^{2}\right)^{l} \widetilde{P}_{k-2 l, n}(\zeta, \omega)\right|^{2} d V_{\widetilde{B}_{p}(r)}(\zeta), \quad \omega \in S .
$$

Then for any $p \geq 1$, we have the following theorem:

Theorem 3.1 (K. Fujita, [2]). The Bergman kernel of $H \mathcal{O}\left(\widetilde{B}_{p}(r)\right)$ is given by

$$
B_{p, r}(z, w)=\sum_{k=0}^{\infty} \sum_{l=0}^{[k / 2]}\left(\beta_{k, l, r}^{n, p}\right)^{-1}\left(z^{2}\right)^{l}\left(\bar{w}^{2}\right)^{l} \widetilde{P}_{k-2 l, n}(z, \bar{w}) .
$$

3.3. Harmonic Bergman kernels. Similarly, for harmonic functions on the $N_{p}$-ball, we define

$$
H \mathcal{O}_{\Delta}\left(\widetilde{B}_{p}(r)\right)=\left\{f \in \mathcal{O}_{\Delta}\left(\widetilde{B}_{p}(r)\right) ; \int_{\widetilde{B}_{p}(r)}|f(w)|^{2} d V_{\widetilde{B}_{p}(r)}(w)<\infty\right\},
$$

and denote by $B_{p, r}^{\Delta}(z, w)$ the harmonic Bergman kernel of $H \mathcal{O}_{\Delta}\left(\widetilde{B}_{p}(r)\right)$; that is, for $f \in H \mathcal{O}_{\Delta}\left(\widetilde{B}_{p}(r)\right)$, we have

$$
f(w)=\int_{\widetilde{B}_{p}(r)} f(z) \overline{B_{p, r}^{\Delta}(z, w)} d V_{\widetilde{B}_{p}(r)}(z), \quad w \in \widetilde{B}_{p}(r) .
$$

Theorem 3.2 (K. Fujita, [3]). The harmonic Bergman kernel of $H \mathcal{O}_{\Delta}\left(\widetilde{B}_{p}(r)\right)$ is given by

$$
B_{p, r}^{\Delta}(z, w)=\sum_{k=0}^{\infty}\left(\beta_{k, 0, r}^{n, p}\right)^{-1} \widetilde{P}_{k, n}(z, w)
$$

3.4. Harmonic continuation. Thanks to Theorem 4.1 in [2] on the Bergman transformation (see Appendix), it is easy to see that the following lemma holds:

Lemma 3.3. Let $w_{0}$ be a point of the boundary $\partial \widetilde{B}_{p}[r]$ of $\widetilde{B}_{p}[r]$. Then as a function of $z, B_{p, r}\left(z, w_{0}\right)$ belongs to $\mathcal{O}\left(\widetilde{B}_{p}(r)\right) \backslash \mathcal{O}\left(\widetilde{B}_{p}[r]\right)$. 
For example, for $p=1,2, \infty$, we can see that $z=w_{0}$ is a singular point of $B_{p, r}\left(z, w_{0}\right)$ from the explicit forms given in Section 3.1.

On the other hand, we have the following theorem (proved in [5] for the 2-dimensional case):

Theorem 3.4. Let $1 \leq p<\infty$ and $f \in H \mathcal{O}_{\Delta}\left(\widetilde{B}_{p}(r)\right)$. Define

$$
F(w)=\int_{\widetilde{B}_{p}(r)} f(z) \overline{B_{p, r}^{\Delta}(z, w)} d V_{\widetilde{B}_{p}(r)}(z) .
$$

Then $F \in \mathcal{O}_{\Delta}\left(\widetilde{B}\left(2^{1 / p} r\right)\right)$ and $F(w)=f(w)$ on $\widetilde{B}_{p}(r)$.

Proof. For $w \in \partial \widetilde{B}_{p}[r]$, put $G_{w}(z)=B_{p, r}^{\Delta}(z, \bar{w})$. Then by Lemma 3.3, as a function of $z, G_{w}(z) \in \mathcal{O}\left(\widetilde{B}_{p}(r)\right)$ and we have

$$
\limsup _{k \rightarrow \infty}\left(\left\|\left(\beta_{k, 0, r}^{n, p}\right)^{-1} \widetilde{P}_{k, n}(\cdot, w)\right\|_{C\left(S_{1}\right)}\right)^{1 / k} \leq 1 /\left(2^{1 / p} r\right)
$$

by Theorem 2.2 in [6] (see Appendix), where $\|\cdot\|_{C\left(S_{1}\right)}$ denotes the supremum norm on $S_{1}$. For $z \in \partial \widetilde{B}_{p}[r]$, since $\widetilde{P}_{k, n}(z, w)=\widetilde{P}_{k, n}(w, z)$, as a function of $w$,

$$
G_{z}(w)=\sum_{k=0}^{\infty}\left(\beta_{k, 0, r}^{n, p}\right)^{-1} \widetilde{P}_{k, n}(z, w) \in \mathcal{O}_{\Delta}\left(\widetilde{B}\left(2^{1 / p} r\right)\right)
$$

by (4) and Theorem 5.2 in [9] (see Appendix). Thus $F(w) \in \mathcal{O}_{\Delta}\left(\widetilde{B}\left(2^{1 / p} r\right)\right)$. For $w \in \widetilde{B}_{p}(r) \subset \widetilde{B}\left(2^{1 / p} r\right)$,

$$
F(w)=\int_{\widetilde{B}_{p}(r)} f(z) \overline{B_{p, r}^{\Delta}(z, w)} d V_{\widetilde{B}_{p}(r)}(z)=f(w) .
$$

More precisely, the mapping $f \mapsto F$ in (3) gives the inverse mapping of the following isomorphisms:

THEOREM 3.5. The restriction mapping $\alpha$ gives the following linear topological isomorphisms:

$$
\alpha: \mathcal{O}_{\Delta}\left(\widetilde{B}\left(2^{1 / p} r\right)\right) \stackrel{\sim}{\longrightarrow} \mathcal{O}_{\Delta}\left(\widetilde{B}_{p}(r)\right), \quad \alpha: \mathcal{O}_{\Delta}\left(\widetilde{B}\left[2^{1 / p} r\right]\right) \stackrel{\sim}{\longrightarrow} \mathcal{O}_{\Delta}\left(\widetilde{B}_{p}[r]\right) .
$$

Proof. If $f \in \mathcal{O}_{\Delta}\left(\widetilde{B}_{p}(r)\right)$, take $\varrho<1$ sufficiently close to 1 and consider the mapping

$$
\beta: f \mapsto F(w)=\int_{\widetilde{B}_{p}(r)} f(\varrho z) \overline{B_{p, r}^{\Delta}(z / \varrho, w)} d V_{\widetilde{B}_{p}(r)}(z), \quad w \in \widetilde{B}_{p}(\varrho r) .
$$

It is easy to see that the right-hand side does not depend on $\varrho$ for $0<\varrho<1$. Since we can take $\varrho$ arbitrarily close to 1 and $\alpha \circ \beta=\beta \circ \alpha=$ id is clear, we have the first isomorphism in (5).

If $f \in \mathcal{O}_{\Delta}\left(\widetilde{B}_{p}[r]\right)$, then take $\varrho>1$ sufficiently close to 1 . Then we have the second isomorphism in (5) by the same argument. 
4. Szegö kernels and harmonic Szegö kernels. We denote by $C\left(\widetilde{B}_{p}[r]\right)$ the space of continuous functions on $\widetilde{B}_{p}[r]$ and by $L^{2}\left(\partial \widetilde{B}_{p}(r), d S_{p, r}\right)$ the space of square integrable functions on $\partial \widetilde{B}_{p}(r)$ with respect to the normalized Lebesgue measure $d S_{p, r}$ on $\partial \widetilde{B}_{p}(r)$. Let $H \mathcal{O}\left(\partial \widetilde{B}_{p}(r)\right)$ be the closure in $L^{2}\left(\partial \widetilde{B}_{p}(r), d S_{p, r}\right)$ of the restrictions to $\partial \widetilde{B}_{p}(r)$ of the elements of $\mathcal{O}\left(\widetilde{B}_{p}(r)\right) \cap C\left(\widetilde{B}_{p}[r]\right):$

$$
H \mathcal{O}\left(\partial \widetilde{B}_{p}(r)\right)=\overline{\left\{f \in \mathcal{O}\left(\widetilde{B}_{p}(r)\right) \cap C\left(\widetilde{B}_{p}[r]\right) ; \int_{\partial \widetilde{B}_{p}(r)}|f(w)|^{2} d S_{p, r}(w)<\infty\right\}} .
$$

We denote by $S_{p, r}(z, w)$ the Szegö kernel on $H \mathcal{O}\left(\partial \widetilde{B}_{p}(r)\right)$ and by $S_{p, r}^{\Delta}(z, w)$ the harmonic Szegö kernel on $H \mathcal{O}_{\Delta}\left(\partial \widetilde{B}_{p}(r)\right)=H \mathcal{O}\left(\partial \widetilde{B}_{p}(r)\right) \cap$ $\mathcal{O}_{\Delta}\left(\widetilde{B}_{p}(r)\right)$. That is, for $f \in H \mathcal{O}\left(\partial \widetilde{B}_{p}(r)\right)$ we have

$$
f(w)=\int_{\partial \widetilde{B}_{p}(r)} f(z) \overline{S_{p, r}(z, w)} d S_{p, r}(z), \quad w \in \widetilde{B}_{p}(r),
$$

and for $f \in H \mathcal{O}_{\Delta}\left(\partial \widetilde{B}_{p}(r)\right)$ we have

$$
f(w)=\int_{\partial \widetilde{B}_{p}(r)} f(z) \overline{S_{p, r}^{\Delta}(z, w)} d S_{p, r}(z), \quad w \in \widetilde{B}_{p}(r) .
$$

For the complex Euclidean ball, the Szegő kernel is known:

$$
S_{2, r}(z, w)=\frac{r^{2 n}}{\left(r^{2}-z \cdot \bar{w}\right)^{n}} .
$$

For the dual Lie ball, E. H. Youssfi obtained the explicit form in [14]:

$$
S_{1, r}(z, w)=\frac{\sum_{k=0}^{[n / 2]} \frac{n ! X^{n-2 k-1} Y^{k}}{(2 k+1) !(n-2 k) !}\left(2(n+1) X-(n-2 k)\left(X^{2}-Y\right)\right)}{(n+2)\left(X^{2}-Y\right)^{n+1}},
$$

where $X=1-z \cdot \bar{w} /\left(2 r^{2}\right)$ and $Y=z^{2} \overline{w^{2}} /\left(4 r^{4}\right)$.

In the case of the Lie ball, the Shilov boundary $\Sigma_{r}$ of $\widetilde{B}(r)$ is known to be

$$
\Sigma_{r}=\left\{e^{i \theta} x \in \mathbb{C}^{n+1} ; \theta \in \mathbb{R}, x \in S_{r}\right\},
$$

which is called the Lie sphere. Thus for the Lie ball, the following kernel has been considered instead of the Szegö kernel: If $f \in \mathcal{O}(\widetilde{B}[r])$, then we have

$$
f(w)=\int_{\Sigma_{r}} f(z) \overline{H_{r}(z, w)} d \Sigma_{r}(z), \quad w \in \widetilde{B}(r),
$$

where $d \Sigma_{r}$ is the normalized Lebesgue measure on the Lie sphere and

$$
H_{r}(z, w)=\frac{r^{2(n+1)}}{\left(r^{4}-2 r^{2} z \cdot \bar{w}+z^{2} \bar{w}^{2}\right)^{(n+1) / 2}}
$$


is the Cauchy-Hua kernel (see Theorem 5.2 in [8]). Its double series expansion is as follows:

$$
H_{r}(z, w)=\sum_{k=0}^{\infty} \sum_{l=0}^{[k / 2]} \frac{N(k-2 l, n)}{r^{2 k}}\left(z^{2}\right)^{l}\left(\bar{w}^{2}\right)^{l} \widetilde{P}_{k-2 l, n}(z, \bar{w}) .
$$

We can also represent the Poisson formula (Formula 2.1) by an integration taken over the Shilov boundary using the Poisson kernel $K_{r}(z, w)$ as follows: For $f \in \mathcal{O}_{\Delta}(\widetilde{B}[r])$, we have

$$
f(w)=\int_{\Sigma_{r}} f(z) \overline{K_{r}(z, w)} d \Sigma_{r}(z), \quad w \in \widetilde{B}(r) .
$$

Put

$$
\alpha_{k, l, r}^{p}=\int_{\partial \widetilde{B}_{p}(r)}\left|\left(\zeta^{2}\right)^{l} \widetilde{P}_{k-2 l, n}(\zeta, \omega)\right|^{2} d S_{p, r}(\zeta), \quad \omega \in S_{1} .
$$

Following [2], we can prove Theorems 4.1 and 4.2 below as in the case of the Bergman kernel. We will omit the proofs.

ThEOREM 4.1. The Szegö kernel of $H \mathcal{O}\left(\partial \widetilde{B}_{p}(r)\right)$ is given by

$$
S_{p, r}(z, \bar{w})=\sum_{k=0}^{\infty} \sum_{l=0}^{[k / 2]}\left(\alpha_{k, l, r}^{p}\right)^{-1}\left(z^{2}\right)^{l}\left(w^{2}\right)^{l} \widetilde{P}_{k-2 l, n}(z, w) .
$$

ThEOREM 4.2. The harmonic Szegö kernel of $H_{\Delta}\left(\partial \widetilde{B}_{p}(r)\right)$ is given by

$$
S_{p, r}^{\Delta}(z, \bar{w})=\sum_{k=0}^{\infty}\left(\alpha_{k, 0, r}^{p}\right)^{-1} \widetilde{P}_{k, n}(z, w) .
$$

\section{2-dimensional case}

5.1. Bergman kernels in $\mathbb{C}^{2}$. For general $n, \beta_{k, l, r}^{n, p}$ have not been calculated except for $p=2, \infty$. But in the case of $n=1, \beta_{k, l, r}^{1, p}$ can be calculated:

$$
\beta_{k, l, r}^{1, p}=\frac{\Gamma\left(\frac{4}{p}+1\right) \Gamma\left(\frac{2 k-2 l+2}{p}\right) \Gamma\left(\frac{2 l+2}{p}\right) 2^{2 k / p} r^{2 k}}{N(k-2 l, 1) \Gamma\left(\frac{2}{p}\right)^{2} \Gamma\left(\frac{2 k+4}{p}+1\right)} .
$$

For $z=\left(z_{1}, z_{2}\right) \in \mathbb{C}^{2}$ we have

$$
N_{p}(z)=\left(\frac{\left|z_{1}+i z_{2}\right|^{p}+\left|z_{1}-i z_{2}\right|^{p}}{2}\right)^{1 / p}, \quad N_{\infty}(z)=L(z)=\max \left|z_{1} \pm i z_{2}\right| .
$$

Thus for $p$ with $1 \leq p<\infty$, the $N_{p}$-norm is equivalent to the $L_{p}$-norm and the Lie norm is equivalent to the supremum norm in $\mathbb{C}^{2}$. J.-D. Park [12] sum- 
med up the infinite sums in explicit form for $p=4,4 / 3$ besides $p=1,2, \infty$. His results are as follows. Put

$$
\begin{aligned}
& f(a, b)=3-6 a+6 b+3 a^{2}-6 a b-b^{2}, \\
& g(a, b)=\left(2-a-b-a^{2}+2 a b-b^{2}\right)(1-a-b) .
\end{aligned}
$$

For $Y_{1}=L(z, \bar{w}) /\left(2^{1 / 2} r^{2}\right)$ and $Y_{2}=M(z, \bar{w}) /\left(2^{1 / 2} r^{2}\right)$, define

$$
\begin{aligned}
F(z, w)= & \frac{Y_{1}\left(\pi+2 \arcsin Y_{1}\right) f\left(Y_{1}^{2}, Y_{2}^{2}\right)}{4\left(1-Y_{1}^{2}\right)^{3 / 2}}+\frac{Y_{2}\left(\pi+2 \arcsin Y_{2}\right) f\left(Y_{2}^{2}, Y_{1}^{2}\right)}{4\left(1-Y_{2}^{2}\right)^{3 / 2}} \\
& +\frac{g\left(Y_{1}^{2}, Y_{2}^{2}\right)}{2\left(1-Y_{1}^{2}\right)\left(1-Y_{2}^{2}\right)}+2 \pi Y_{1} Y_{2} .
\end{aligned}
$$

Then

$$
B_{4, r}(z, w)=\frac{r^{12} F(z, w)}{\left(r^{4}-2(z \cdot \bar{w})^{2}+z^{2} \bar{w}^{2}\right)^{3}}=\frac{r^{12} F(z, w)}{\left(r^{4}-N_{4}^{4}(z, \bar{w})\right)^{3}},
$$

where $N_{4}^{4}(z, w)=\left(L(z, w)^{2}+M(z, w)^{2}\right) / 2$. Note that $N_{4}^{4}(z, \bar{z})=N_{4}(z)^{4}$.

Take a branch of $L(z, \bar{w})^{1 / 3}$ and $M(z, \bar{w})^{1 / 3}$, and put $\omega=e^{2 \pi i / 3}$,

$$
\begin{gathered}
Y_{1}\left(j_{1}\right)=\omega^{j_{1}} \frac{L(z, \bar{w})^{1 / 3}}{2^{1 / 2} r^{2 / 3}}, \quad Y_{2}\left(j_{2}\right)=\omega^{j_{2}} \frac{M(z, \bar{w})^{1 / 3}}{2^{1 / 2} r^{2 / 3}}, \\
F_{j_{1}, j_{2}}(z, w)=\frac{F\left(Y_{1}\left(j_{1}\right), Y_{2}\left(j_{2}\right)\right)}{Y_{1}\left(j_{1}\right)^{2} Y_{2}\left(j_{2}\right)^{2}} .
\end{gathered}
$$

Then applying Theorem 1 of Bell [1], we have

$$
B_{4 / 3, r}(z, w)=\frac{1}{216} \sum_{j_{1}=1}^{3} \sum_{j_{2}=1}^{3} \frac{F_{j_{1}, j_{2}}(z, w)}{\left(1-Y_{1}\left(j_{1}\right)^{2}-Y_{2}\left(j_{2}\right)^{2}\right)^{3}} .
$$

5.2. Harmonic Bergman kernels in $\mathbb{C}^{2}$. For the 2-dimensional $N_{p}$-balls we gave the harmonic Bergman kernels in explicit form for $p=1,2, \infty$ in [3]. Put

$$
\begin{aligned}
P(s, t)= & 1+2 s-24 t+60 s t+4 t^{2} \\
& +18 s t^{2}-80 s^{2} t-4 t^{3}+8 s t^{3}-24 s^{2} t^{2}+40 s^{3} t-t^{4} .
\end{aligned}
$$

Then

$$
\begin{aligned}
B_{1, r, \Delta}(z, w) & =\frac{r^{16} P\left(z \cdot \bar{w} /\left(4 r^{2}\right), z^{2} \bar{w}^{2} /\left(4 r^{4}\right)\right)}{\left(r^{2}-L(z, \bar{w}) / 4\right)^{4}\left(r^{2}-M(z, \bar{w}) / 4\right)^{4}} \\
& =\frac{r^{16} P\left(z \cdot \bar{w} /\left(4 r^{2}\right), z^{2} \bar{w}^{2} /\left(4 r^{4}\right)\right)}{\left(r^{4}-r^{2} z \cdot \bar{w} / 2+z^{2} \bar{w}^{2} / 16\right)^{4}}
\end{aligned}
$$




$$
\begin{aligned}
& B_{2, r, \Delta}(z, w) \\
& =\frac{r^{6}\left(2 r^{6}-3 r^{4} z \cdot \bar{w}+3 r^{4}(z \cdot \bar{w})^{2}-3 r^{2} z^{2} \bar{w}^{2} / 2-(z \cdot \bar{w})^{3}+3 z \cdot \bar{w} z^{2} \bar{w}^{2} / 4\right)}{\left(r^{2}-L(z, \bar{w}) / 2\right)^{3}\left(r^{2}-M(z, \bar{w}) / 2\right)^{3}}-1 \\
& =\frac{r^{6}\left(2 r^{6}-3 r^{4} z \cdot \bar{w}+3 r^{2}(z \cdot \bar{w})^{2}-3 r^{2} z^{2} \bar{w}^{2} / 2-(z \cdot \bar{w})^{3}+3 z \cdot \bar{w} z^{2} \bar{w}^{2} / 4\right)}{\left(r^{4}-r^{2} z \cdot \bar{w}+z^{2} \bar{w}^{2} / 4\right)^{3}}-1, \\
& B_{r, \Delta}(z, w)=\frac{r^{8}-z^{2} \bar{w}^{2}\left(4 r^{4}-4 r^{2} z \cdot \bar{w}+z^{2} \bar{w}^{2}\right)}{\left(r^{2}-L(z, \bar{w})\right)^{2}\left(r^{2}-M(z, \bar{w})\right)^{2}} \\
& =\frac{r^{8}-z^{2} \bar{w}^{2}\left(4 r^{4}-4 r^{2} z \cdot \bar{w}+z^{2} \bar{w}^{2}\right)}{\left(r^{4}-2 r^{2} z \cdot \bar{w}+z^{2} \bar{w}^{2}\right)^{2}} .
\end{aligned}
$$

Note that the denominators of the kernels are consistent with Theorem 3.4.

By the work of J.-D. Park [12], we have

$$
G_{4}(X)=\sum_{k=0}^{\infty} \frac{\sqrt{\pi} \Gamma(k / 2+2)}{\Gamma((k+1) / 2)} X^{k}=\frac{3 X(\pi+2 \arcsin X)}{4\left(1-X^{2}\right)^{5 / 2}}+\frac{X^{2}+2}{2\left(1-X^{2}\right)^{2}} .
$$

Thus putting $X_{1}=L(z, \bar{w}) /\left(2^{1 / 4} r\right)^{2}, X_{2}=M(z, \bar{w}) /\left(2^{1 / 4} r\right)^{2}$, we have

$$
\begin{aligned}
B_{4, r, \Delta}(z, w)= & \frac{3 X_{1}\left(\pi+2 \arcsin X_{1}\right)}{4\left(1-X_{1}^{2}\right)^{5 / 2}} \\
& +\frac{X_{1}^{2}+2}{2\left(1-X_{1}^{2}\right)^{2}}+\frac{3 X_{2}\left(\pi+2 \arcsin X_{2}\right)}{4\left(1-X_{2}^{2}\right)^{5 / 2}}+\frac{X_{2}^{2}+2}{2\left(1-X_{2}^{2}\right)^{2}}-1 .
\end{aligned}
$$

Next, consider the infinite sum

$$
G_{4 / 3}(X)=\frac{\sqrt{\pi}}{12} \sum_{k=0}^{\infty} \frac{\Gamma(3(k+1) / 2+5 / 2)}{\Gamma(3(k+1) / 2)} X^{k}
$$

Put

$$
F(X)=\frac{15 X^{2}(\pi+2 \arcsin X)}{4\left(1-X^{2}\right)^{7 / 2}}+\frac{X\left(-2 X^{4}+9 X^{2}+8\right)}{2\left(1-X^{2}\right)^{3}} .
$$

Then employing an idea considered in Theorem 1 of Bell [1] for Bergman kernels, we can sum up $G_{4 / 3}(X)$ in explicit form as follows: for $\omega=e^{2 \pi i / 3}$,

$$
G_{4 / 3}(X)=\frac{1}{72 X}\left(F\left(\omega X^{1 / 3}\right)+F\left(\omega^{2} X^{1 / 3}\right)+F\left(X^{1 / 3}\right)\right) .
$$

Thus we have

$$
B_{4 / 3, r, \Delta}(z, w)=G_{4 / 3}\left(L(z, \bar{w}) /\left(2^{3 / 2} r^{2}\right)\right)+G_{4 / 3}\left(M(z, \bar{w}) /\left(2^{3 / 2} r^{2}\right)\right)-1 .
$$

5.3. Szegö kernels and harmonic Szegö kernels in $\mathbb{C}^{2}$. For the 2-dimensional case, the coefficients $\alpha_{k, l, r}^{p}$ can be calculated and Theorems 4.1 and 4.2 read as follows: 
TheOREM 5.1. In $\mathbb{C}^{2}$, the Szegö kernel $S_{p, r}(z, w)$ is given as follows:

$$
\begin{aligned}
S_{p, r}(z, w) & =\sum_{k=0}^{\infty} \sum_{l=0}^{[k / 2]} \frac{N(k-2 l, 1) \Gamma(2 / p)^{2} \Gamma((2 k+4) / p)\left(z^{2}\right)^{l}\left(\bar{w}^{2}\right)^{l} \widetilde{T}_{k-2 l}(z, \bar{w})}{\Gamma(4 / p) \Gamma((2 k-2 l+2) / p) \Gamma((2 l+2) / p) 2^{2 k / p} r^{2 k}} \\
& =\sum_{k=0}^{\infty} \sum_{l=0}^{\infty} \frac{\Gamma(2 / p)^{2} \Gamma((2 k+2 l+4) / p) L(z, \bar{w})^{k} M(z, \bar{w})^{l}}{\Gamma(4 / p) \Gamma((2 k+2) / p) \Gamma((2 l+2) / p) 2^{(2 k+2 l) / p} r^{2 k+2 l}} .
\end{aligned}
$$

By this theorem, (7) and (6), we have

$$
\begin{aligned}
S_{1, r}(z, w) & =\sum_{k=0}^{\infty} \sum_{l=0}^{\infty} \frac{\Gamma(2 k+2 l+4) L(z, \bar{w})^{k} M(z, \bar{w})^{l}}{6 \Gamma(2 k+2) \Gamma(2 l+2) 2^{2 k+2 l} r^{2 k+2 l}} \\
& =\frac{r^{8}\left(3 r^{8}-r^{6} z \cdot \bar{w}-r^{4}(z \cdot \bar{w})^{2} / 4+\left(z^{2} \bar{w}^{2}\right)^{2} / 16\right)}{3\left(r^{8}-r^{6} z \cdot \bar{w}+r^{4}(z \cdot \bar{w})^{2} / 4-\left(z^{2} \bar{w}^{2}\right)^{2} / 16\right)^{2}}, \\
S_{2, r}(z, w) & =\sum_{k=0}^{\infty} \sum_{l=0}^{\infty} \frac{\Gamma(k+l+2) L(z, \bar{w})^{k} M(z, \bar{w})^{l}}{\Gamma(k+1) \Gamma(l+1) 2^{k+l} r^{2 k+2 l}} \\
& =\frac{r^{4}}{\left(r^{2}-z \cdot \bar{w}\right)^{2}}=\frac{r^{4}}{\left(r^{2}-(L(z, \bar{w})+M(z, \bar{w})) / 2\right)^{2}} .
\end{aligned}
$$

By [12], for $p=4 / 3,4$ we have

$$
\begin{aligned}
& S_{4, r}(z, w)=\sum_{k=0}^{\infty} \sum_{l=0}^{\infty} \frac{\pi \Gamma((k+l+2) / 2) L(z, \bar{w})^{k} M(z, \bar{w})^{l}}{\Gamma((k+1) / 2) \Gamma((l+1) / 2) 2^{(k+l) / 2} r^{2 k+2 l}} \\
& =\frac{\pi r^{4} z^{2} \bar{w}^{2}}{2\left(r^{4}-2(z \cdot \bar{w})^{2}+z^{2} \bar{w}^{2}\right)^{2}}+\frac{r^{4}}{\left(r^{4}-2(z \cdot \bar{w})^{2}+z^{2} \bar{w}^{2}\right)} \\
& +\frac{r^{4} L(z, \bar{w})\left(r^{4}-2 z \bar{w} \sqrt{(z \cdot \bar{w})^{2}-z^{2} \bar{w}^{2}}\right)\left(\pi+2 \arcsin \left(L(z, \bar{w}) /\left(2^{1 / 2} r^{2}\right)\right)\right)}{2\left(r^{4}-2(z \cdot \bar{w})^{2}+z^{2} \bar{w}^{2}\right)^{2}\left(2 r^{4}-L(z, \bar{w})^{2}\right)^{1 / 2}} \\
& +\frac{r^{4} M(z, \bar{w})\left(r^{4}+2 z \bar{w} \sqrt{(z \cdot \bar{w})^{2}-z^{2} \bar{w}^{2}}\right)\left(\pi+2 \arcsin \left(M(z, \bar{w}) /\left(2^{1 / 2} r^{2}\right)\right)\right)}{2\left(r^{4}-2(z \cdot \bar{w})^{2}+z^{2} \bar{w}^{2}\right)^{2}\left(2 r^{4}-M(z, \bar{w})^{2}\right)^{1 / 2}} \\
& S_{4 / 3, r}(z, w)=\sum_{k=0}^{\infty} \sum_{l=0}^{\infty} \frac{\pi \Gamma(3(k+l+2) / 2) L(z, \bar{w})^{k} M(z, \bar{w})^{l}}{8 \Gamma(3(k+1) / 2) \Gamma(3(l+1) / 2) 2^{3(k+l) / 2} r^{2 k+2 l}} \\
& =\frac{1}{72} \sum_{j_{1}=1}^{3} \sum_{j_{2}=1}^{3} \frac{S_{4, r}\left(\omega^{j_{1}}\left(L(z, \bar{w}) /\left(2^{1 / 2} r^{2}\right)\right)^{1 / 3}, \omega^{j_{2}}\left(M(z, \bar{w}) /\left(2^{1 / 2} r^{2}\right)\right)^{1 / 3}\right)}{\omega^{j_{1}} \omega^{j_{2}}\left(L(z, \bar{w}) /\left(2^{1 / 2} r^{2}\right)\right)^{2 / 3}\left(M(z, \bar{w}) /\left(2^{1 / 2} r^{2}\right)\right)^{2 / 3}} .
\end{aligned}
$$

Furthermore, for the two-dimensional Lie ball we have the following explicit form: 


$$
\begin{aligned}
S_{\infty, r}(z, w)= & \sum_{k=0}^{\infty} \sum_{l=0}^{\infty} \frac{2(k+1)(l+1)}{(k+l+2)} \frac{L(z, \bar{w})^{k} M(z, \bar{w})^{l}}{r^{2 k+2 l}} \\
= & 2 \frac{r^{2} L(z, \bar{w})+r^{2} M(z, \bar{w})-2 L(z, \bar{w}) M(z, \bar{w})}{\left(1-L(z, \bar{w}) / r^{2}\right)\left(1-M(z, \bar{w}) / r^{2}\right)(L(z, \bar{w})-M(z, \bar{w}))^{2}} \\
& -2 \frac{L(z, \bar{w}) / r^{2}+M(z, \bar{w}) / r^{2}}{(L(z, \bar{w})-M(z, \bar{w}))^{3} / r^{6}} \log \frac{r^{2}-L(z, \bar{w})}{r^{2}-M(z, \bar{w})} \\
= & \frac{4 r^{4}\left(r^{2} z \cdot \bar{w}-z^{2} \bar{w}^{2}\right)}{\left(r^{4}-2 r^{2} z \cdot \bar{w}+z^{2} \overline{w^{2}}\right)\left((z \cdot \bar{w})^{2}-z^{2} \bar{w}^{2}\right)} \\
& -\frac{2 r^{4} z \cdot \bar{w}}{\left((z \cdot \bar{w})^{2}-z^{2} \bar{w}^{2}\right)^{3 / 2}} \log \frac{r^{2}-z \cdot \bar{w}-\left((z \cdot \bar{w})^{2}-z^{2} \overline{w^{2}}\right)^{1 / 2}}{r^{2}-z \cdot \bar{w}+\left((z \cdot \bar{w})^{2}-z^{2} \overline{w^{2}}\right)^{1 / 2}} .
\end{aligned}
$$

Recall that the Cauchy-Hua kernel related to the Shilov boundary of the Lie ball (Lie sphere) mentioned in Section 4 is simpler:

$$
H_{r}(z, w)=\frac{r^{4}}{r^{4}-2 r^{2} z \cdot \bar{w}+z^{2} \bar{w}^{2}} .
$$

THEOREM 5.2. In $\mathbb{C}^{2}$, the harmonic Szegö kernel $S_{p, r}^{\Delta}(z, w)$ is given as follows:

$$
\begin{aligned}
S_{p, r}^{\Delta}(z, w) & =\sum_{k=0}^{\infty} \frac{N(k, 1) \Gamma(2 / p) \Gamma((2 k+4) / p)}{\Gamma(4 / p) \Gamma((2 k+2) / p) 2^{2 k / p} r^{2 k}} \widetilde{T}_{k}(z, \bar{w}) \\
& =\sum_{k=0}^{\infty} \frac{\Gamma(2 / p) \Gamma((2 k+4) / p)}{\Gamma(4 / p) \Gamma((2 k+2) / p) 2^{2 k / p} r^{2 k}}\left(L(z, \bar{w})^{k}+M(z, \bar{w})^{k}\right)-1 .
\end{aligned}
$$

For harmonic Szegő kernels, we can also sum up the infinite series in explicit form for $p=1,4 / 3,2,4$ and $p=\infty$ :

$$
\begin{aligned}
S_{1, r}^{\Delta}(z, w)= & \sum_{k=0}^{\infty} \frac{\Gamma(2 k+4)}{6 \Gamma(2 k+2) 2^{2 k} r^{2 k}}\left(L(z, \bar{w})^{k}+M(z, \bar{w})^{k}\right)-1 \\
= & \frac{r^{4} L(z, \bar{w})\left(r^{2}-M(z, \bar{w}) / 4\right)^{3}+r^{4} M(z, \bar{w})\left(r^{2}-L(z, \bar{w}) / 4\right)^{3}}{3\left(r^{4}-r^{2} z \cdot \bar{w}+z^{2} \bar{w}^{2} / 16\right)^{3}} \\
& +\frac{r^{2} L(z, \bar{w})\left(r^{2}-M(z, \bar{w}) / 4\right)^{2}+r^{2} M(z, \bar{w})\left(r^{2}-L(z, \bar{w}) / 4\right)^{2}}{4\left(r^{4}-r^{2} z \cdot \bar{w}+z^{2} \bar{w}^{2} / 16\right)^{2}}-1, \\
S_{2, r}^{\Delta}(z, w)= & \sum_{k=0}^{\infty} \frac{k+1}{2^{k} r^{2 k}}\left(L(z, \bar{w})^{k}+M(z, \bar{w})^{k}\right)-1 \\
= & \frac{r^{8}-r^{4} z^{2} \bar{w}^{2}+\left(z^{2} \bar{w}^{2}\right)^{2} / 16-r^{2} z \cdot \bar{w} z^{2} \bar{w}^{2} / 2}{\left(r^{4}-r^{2} z \cdot \bar{w}+z^{2} \bar{w}^{2} / 4\right)^{2}},
\end{aligned}
$$




$$
\begin{aligned}
S_{4, r}^{\Delta}(z, w) & =\sum_{k=0}^{\infty} \frac{\sqrt{\pi} \Gamma((k+2) / 2)}{\Gamma((k+1) / 2) 2^{k / 2} r^{2 k}}\left(L(z, \bar{w})^{k}+M(z, \bar{w})^{k}\right)-1 \\
& =F_{4}\left(L(z, \bar{w}) /\left(2^{1 / 2} r^{2}\right)\right)+F_{4}\left(M(z, \bar{w}) /\left(2^{1 / 2} r^{2}\right)\right)-1
\end{aligned}
$$

where $F_{4}(x)=1 /\left(1-x^{2}\right)+x\left(\pi+2 \arcsin x^{2}\right) /\left(2\left(1-x^{2}\right)^{3 / 2}\right)$;

$$
\begin{aligned}
S_{4 / 3, r}^{\Delta}(z, w) & =\sum_{k=0}^{\infty} \frac{\sqrt{\pi} \Gamma(3(k+2) / 2)}{4 \Gamma(3(k+1) / 2) 2^{3 k / 2} r^{2 k}}\left(L(z, \bar{w})^{k}+M(z, \bar{w})^{k}\right)-1 \\
& =F_{4 / 3}\left(L(z, \bar{w}) /\left(2^{3 / 2} r^{2}\right)\right)+F_{4 / 3}\left(M(z, \bar{w}) /\left(2^{3 / 2} r^{2}\right)\right)-1,
\end{aligned}
$$

where

$$
F_{4 / 3}(x)=\frac{2}{x^{2}\left(1-x^{2}\right)^{2}}+\frac{2 x\left(1+x^{2}\right)^{1 / 2}}{\left(1-x^{2}\right)^{2}}+\frac{3\left(\pi+2 \arcsin x^{2}\right)}{2 x\left(1-x^{2}\right)^{5 / 2}}
$$

and

$$
\begin{aligned}
S_{\infty, r}^{\Delta}(z, w)= & \sum_{k=0}^{\infty} \frac{N(k, 1) 2(k+1)}{(k+2) r^{2 k}} \widetilde{T}_{k}(z, \bar{w}) \\
= & \frac{2 r^{4}}{\left(r^{2}-L(z, \bar{w})\right) L(z, \bar{w})}+\frac{2 r^{4}}{\left(r^{2}-M(z, \bar{w})\right) M(z, \bar{w})} \\
& +\frac{2 r^{4} \log \left(1-L(z, \bar{w}) / r^{2}\right)}{L(z, \bar{w})^{2}}+\frac{2 r^{4} \log \left(1-M(z, \bar{w}) / r^{2}\right)}{M(z, \bar{w})^{2}}-1 \\
= & 4 r^{4} \frac{r^{2} z \cdot \bar{w}-2(z \cdot \bar{w})^{2}+z^{2} \bar{w}^{2}}{\left(r^{4}-2 r^{2} z \cdot \bar{w}+z^{2} \bar{w}^{2}\right) z^{2} \bar{w}^{2}}-1 \\
+ & 2 r^{4} \frac{M(z, \bar{w})^{2} \log \left(1-L(z, \bar{w}) / r^{2}\right)+L(z, \bar{w})^{2} \log \left(1-M(z, \bar{w}) / r^{2}\right)}{\left(z^{2} \bar{w}^{2}\right)^{2}} .
\end{aligned}
$$

Recall that the Poisson kernel related to the Shilov boundary of the Lie ball mentioned in Section 4 is simpler:

$$
K_{r}(z, w)=\frac{r^{4}-z^{2} \bar{w}^{2}}{r^{4}-2 r^{2} z \cdot \bar{w}+z^{2} \bar{w}^{2}} .
$$

6. Appendix. In this section we quote some theorems to which we referred in the proofs.

We denote by $\mathcal{O}^{\prime}\left(\widetilde{B}_{p}(r)\right)$ and $\mathcal{O}^{\prime}\left(\widetilde{B}_{p}[r]\right)$ the dual spaces of $\mathcal{O}\left(\widetilde{B}_{p}(r)\right)$ and $\mathcal{O}\left(\widetilde{B}_{p}[r]\right)$, respectively. For $T \in \mathcal{O}^{\prime}\left(\widetilde{B}_{p}[r]\right)$ define $\mathbf{B}_{r}^{p} T(w)=\left\langle T_{z}, B_{r}^{p}(z, \bar{w})\right\rangle$, where $\langle$,$\rangle is the canonical inner product on \mathcal{O}^{\prime}\left(\widetilde{B}_{p}[r]\right) \times \mathcal{O}\left(\widetilde{B}_{p}[r]\right)$. The bilinear form

$$
\langle f, g\rangle_{\widetilde{B}_{p}[r]}=\int_{\widetilde{B}_{p}[r]} f(\varrho z) g(\bar{z} / \varrho) d V_{\widetilde{B}_{p}(r)}(z)
$$

is well-defined for $f \in \mathcal{O}\left(\widetilde{B}_{p}(r)\right)$ and $g \in \mathcal{O}\left(\widetilde{B}_{p}[r]\right)$. 
6.1 (Theorem 4.1 in [2]). The Bergman transformation $\mathbf{B}_{r}^{p}: T \mapsto \mathbf{B}_{r}^{p} T(w)$ establishes the following topological linear isomorphisms:

$$
\mathbf{B}_{r}^{p}: \mathcal{O}^{\prime}\left(\widetilde{B}_{p}[r]\right) \stackrel{\sim}{\longrightarrow} \mathcal{O}\left(\widetilde{B}_{p}(r)\right), \quad \mathbf{B}_{r}^{p}: \mathcal{O}^{\prime}\left(\widetilde{B}_{p}(r)\right) \stackrel{\sim}{\longrightarrow} \mathcal{O}\left(\widetilde{B}_{p}[r]\right) .
$$

We have $\langle T, f\rangle=\left\langle f, \mathbf{B}_{r}^{p} T\right\rangle_{\widetilde{B}_{p}[r]}$ for $T \in \mathcal{O}^{\prime}\left(\widetilde{B}_{p}(r)\right)$ and $f \in \mathcal{O}\left(\widetilde{B}_{p}(r)\right)$, as well as for $T \in \mathcal{O}^{\prime}\left(\widetilde{B}_{p}[r]\right)$ and $f \in \mathcal{O}\left(\widetilde{B}_{p}[r]\right)$.

6.2 (Theorem 2.2 in $[6]$ ). Let $f_{k-2 l}$ be the homogeneous harmonic polynomial of degree $k-2 l$. Then

$$
\begin{aligned}
& \sum_{k=0}^{\infty} \sum_{l=0}^{[k / 2]}\left(z^{2}\right)^{l} f_{k-2 l}(z) \in \mathcal{O}\left(\widetilde{B}_{p}(r)\right) \\
& \Leftrightarrow \limsup _{k \rightarrow \infty}\left(\left(\frac{l !(k-l) !}{k !}\right)^{1 / p}\left\|f_{k-2 l}\right\|_{C\left(S_{1}\right)}\right)^{1 / k} \leq \frac{1}{2^{1 / p}} .
\end{aligned}
$$

6.3 (Theorem 5.2 in [9]). Let $f_{k}$ be the homogeneous harmonic polynomial of degree $k$. Then

$$
\sum_{k=0}^{\infty} f_{k}(z) \in \mathcal{O}_{\Delta}(\widetilde{B}(r)) \Leftrightarrow \limsup _{k \rightarrow \infty}\left(\left\|f_{k}\right\|_{C\left(S_{1}\right)}\right)^{1 / k} \leq 1 / r .
$$

\section{References}

[1] S. Bell, The Bergman kernel function and proper holomorphic mapppings, Trans. Amer. Math. Soc. 270 (1982), 685-691.

[2] K. Fujita, Bergman transformation for analytic functionals on some balls, in: Microlocal Analysis and Complex Fourier Analysis, World Sci., 2002, 81-98.

[3] - , Harmonic Bergman kernel for some balls, Univ. Iagel. Acta Math. 41 (2003), $225-234$.

[4] -, Bergman kernel for the two-dimensional balls, Complex Variables Theory Appl. 49 (2004), 215-225.

[5] - Topics on the Bergman kernel for some balls, in: Proc. 5th Internat. ISAAC Congress, to appear.

[6] K. Fujita and M. Morimoto, On the double series expansion of holomorphic functions, J. Math. Anal. Appl. 272 (2002), 335-348.

[7] L. K. Hua, Harmonic Analysis of Functions of Several Complex Variables in Classical Domains, Izdat. Inostr. Lit., Moscow, 1959 (in Russian); English transl.: Transl. Math. Monogr. 6, Amer. Math. Soc., Providence, RI, 1979.

[8] M. Morimoto, Analytic functionals on the Lie sphere, Tokyo J. Math. 3 (1980), $1-35$.

[9] - Analytic functionals on the sphere and their Fourier-Borel transformations, in: Complex Analysis, Banach Center Publ. 11, PWN-Polish Sci. Publ., Warszawa, 1983, 223-250.

[10] M. Morimoto and K. Fujita, Between Lie norm and dual Lie norm, Tokyo J. Math. 24 (2001), 499-507. 
[11] K. Oeljklaus, P. Pflug and E. H. Youssfi, The Bergman kernels of the minimal ball and applications, Ann. Inst. Fourier (Grenoble) 47 (1997), 915-928.

[12] J.-D. Park, Explicit computations of the Bergman kernel and Toeplitz products on the Bergman spaces, doctoral thesis, Seoul National Univ., 2004.

[13] J. Siciak, Holomorphic continuation of harmonic functions, Ann. Polon. Math. 29 (1974), 67-73.

[14] E. H. Youssfi, Proper holomorphic liftings and new formulas for the Bergman and Szegő kernels, Studia Math. 152 (2002), 161-186.

Faculty of Culture and Education

Saga University

Saga, 840-8502, Japan

E-mail: keiko@cc.saga-u.ac.jp

Received 30.11.2006

and in final form 15.2.2007 